$\operatorname{MITP} / 13-050$

\title{
New chiral lattice actions of the Boriçi-Creutz type
}

\author{
Stefano Capitani* \\ Institut für Kernphysik and HIM (Helmholtz-Institut Mainz), \\ University of Mainz, Johann-Joachim-Becher-Weg 45, D-55099 Mainz, Germany
}

\begin{abstract}
We generalize the Boriçi-Creutz action in such a way that the position of the second zero and the direction which breaks the hypercubic symmetry can be arbitrarily chosen, and the action has still the correct continuum limit. Minimal doubling is guaranteed if the distance between the two zeros does not become too large. Special values of this distance could turn out to be particularly convenient for efficient numerical simulations of minimally doubled fermions.
\end{abstract}

November 2013

*capitan@kph.uni-mainz.de 


\section{Introduction}

Graphene-inspired Boriçi-Creutz fermions [1,2,3,4] have sparked off a few years ago a revival of minimally doubled actions (a recent overview is in [5]). With these simple lattice formulations Monte Carlo simulations of two degenerate flavors preserving chiral symmetry for any finite lattice spacing $a$ can be carried out. Two flavors is the minimum value allowed by the Nielsen-Ninomiya theorem if one wants to keep an exact continuous chiral symmetry (of the standard type, i.e. not Ginsparg-Wilson) and also maintain other convenient field-theoretical properties like locality and unitarity.

These chiral fermionic formulations can be still kept ultralocal (like Wilson fermions), and since they contain only nearest-neighbor interactions, they are cheap and easy to simulate. Their formalism is also quite simple, and one can construct conserved axial currents which have a simple and compact expression.

Minimally doubled fermions have the same kind of $U(1)$ chiral symmetry as staggered fermions, and are slightly more expensive compared with them. However, having 2 flavors instead of 4 they do not require uncontrolled extrapolations to 2 physical light flavors, and so they are ideal for $N_{f}=2$ simulations. One also avoids the complicated intertwining of spin and flavor of staggered fermions. 11

As they are much cheaper than Ginsparg-Wilson fermions, minimally doubled fermions can be very convenient for vector-like theories like QCD. Moreover, they might be very practical for simulations of lattice QCD at finite temperature, where staggered fermions are extensively used.

Boriçi-Creutz fermions have a special place among minimally doubled fermions, not only because they have sparked off the revival of this class of ultralocal chiral formulations, but also for their particular construction which has arisen from investigations of the properties of electrons in graphene. Boriçi-Creutz fermions are an instructive example of models based on spinless fermions hopping on a lattice, in which the low-energy excitations come out at the end to carry half-integer spin.2 On the lattice strictly speaking the spin-statistics theorem is not valid (as relativistic invariance is broken), and the emergence of spin from spinless particles has been put forward by Creutz and recently discussed in [6]. How the spin arises is dictated by the topological behavior of the action in momentum space. It is quite interesting to see how the topological protection from additive mass renormalization works, and also how the topological properties of the action constrain the fermionic flavors to appear only in an even number, giving so an intriguing picture of the workings of the Nielsen-Ninomiya theorem.

Together with Boriçi-Creutz fermions, another simple realization of minimally doubled fermions known as Karsten-Wilczek fermions [7,8] has also been studied in some detail in the last few years. The minimal doubling of the latter formulation comes out through a more "standard" mechanism, more similar to that of Wilson fermions.

\footnotetext{
${ }^{1}$ Very recently a minimally doubled staggered action has been presented by Creutz in [6]. This action contains hermitian as well as anti-hermitian parts, and breaks hypercubic symmetry like all other minimally doubled actions found so far (in this case it is the temporal direction which is treated in a different way).

${ }^{2}$ Another example is given by the above mentioned minimally doubled staggered fermions of Creutz [6], constructed out of a lattice of spinless fermions subjected to a constant magnetic field.
} 
Many properties of these two actions have been studied and more deeply understood in 9,10,11,12, where the Boriçi-Creutz and Karsten-Wilczek fermions have been shown to form a fully consistent renormalized quantum field theory.

The standard Karsten-Wilczek action, which has the two zeros at a fixed location, has been recently generalized in such a way that an arbitrary distance between its two zeros can be chosen [13,14,15]. Achieving something similar for the standard BoriçiCreutz action as well, where the position of the second zero is also constrained to a specific location, has turned out to be less straightforward. In this article we present the first successful formulation of a generalized Boriçi-Creutz action with variable distance between the two zeros. Moreover, the direction of hypercubic breaking can also be chosen at will.

In general it can be convenient to have at hand minimally doubled actions where the distance between the two poles of the quark propagator can be arbitrarily varied. Special values of this distance could also provide actions which are more advantageous for numerical simulations, in that for instance they minimize some artefacts specific to these formulations. Here we have a $U(1)$ chiral symmetry instead of the continuum $S U(2)$, and only the neutral pion remains as a Goldstone boson, while the charged pions will have a higher mass. The effective amount of important physical quantities such as the mass difference between the $\pi^{ \pm}$and the $\pi^{0}$, or of mass splittings within otherwise degenerate multiplets, could turn out to be rather small for a few of these actions and not so small for all other ones. Then, having the possibility of moving the distance between the two poles could be useful in order to minimize in the continuum limit the effects (among others) coming from having only a $U(1)$ chiral symmetry.

Thus, it is always useful to possess as many different minimally doubled actions as possible, and keep on trying to construct new ones. Some particular actions could turn out to have better theoretical or practical properties, and be particularly advantageous for lattice simulations of chiral fermions.

This article is organized as follows. In Sect. 2 we recall the form of the standard Boriçi-Creutz action and present its generalization, which allows an arbitrary position of the second zero, while in Sect. 3 we explain how one can arrive at this generalized action. Then in Sect. 4 we give the new Dirac matrices that have to be introduced, while in Sect. 5] we discuss which choices of the position of the second zero allow minimal doubling to be preserved, before giving an outlook in Sect. 6. Finally, in Appendix A we provide a few derivations of important properties of the zeros, which make use of the trace equations.

\section{The generalized action}

The work of Creutz [1,3] and Boriçi [2,4] has led to the formulation of a fermionic action whose Dirac operator in momentum space is given, in the free case, by

$$
\begin{aligned}
D^{B C}(p) & =\frac{i}{a} \sum_{\mu}\left(\gamma_{\mu} \sin a p_{\mu}-\gamma_{\mu}^{\prime}\left(1-\cos a p_{\mu}\right)\right)+m_{0} \\
& =\frac{i}{a} \sum_{\mu}\left(\gamma_{\mu} \sin a p_{\mu}+\gamma_{\mu}^{\prime} \cos a p_{\mu}\right)-\frac{2 i \Gamma}{a}+m_{0}
\end{aligned}
$$


where

$$
\gamma_{\mu}^{\prime}=\Gamma \gamma_{\mu} \Gamma
$$

and

$$
\Gamma=\frac{1}{2} \sum_{\mu} \gamma_{\mu}=\frac{1}{2} \sum_{\mu} \gamma_{\mu}^{\prime}
$$

with $\Gamma^{2}=1$. This action vanishes at $a p_{\mu}=(0,0,0,0)$ and $a p_{\mu}=(\pi / 2, \pi / 2, \pi / 2, \pi / 2)$ and describes two fermion species of opposite chirality. The two zeros determine the special direction which breaks the hypercubic symmetry, which is in this case a major hypercubic diagonal. This special direction is also encoded in the expression of the matrix $\Gamma$.

We generalize here the Boriçi-Creutz action in such a way that the second zero can be moved to an arbitrary position $a p_{\mu}=\alpha_{\mu}$, with $-\pi<\alpha_{\mu}<\pi$ (but $\alpha_{\mu} \neq 0$ ). The components of $\alpha_{\mu}$ do not need to be equal, and actually they can even be all different from one another. Thus, the direction of hypercubic breaking can be arbitrarily chosen. However, as we will understand later, this direction can never exactly correspond to one of the $p_{\mu}$ axes.

The Dirac operator of the generalized Boriçi-Creutz action that we have found is given in position space by

$$
D=\frac{1}{2}\left\{\sum_{\mu=1}^{4} \gamma_{\mu}\left(\nabla_{\mu}+\nabla_{\mu}^{*}\right)+i a \sum_{\mu=1}^{4}\left(\gamma_{\mu} \cot \alpha_{\mu}+\gamma_{\mu}^{\prime} \csc \alpha_{\mu}\right) \nabla_{\mu}^{*} \nabla_{\mu}\right\}+m_{0},
$$

where

$$
\nabla_{\mu} \psi(x)=\frac{U_{\mu}(x) \psi(x+a \widehat{\mu})-\psi(x)}{a}
$$

is a lattice discretization of the covariant derivative, and $\gamma^{\prime}$ is another set of Dirac matrices, which will be specified later. After expanding the covariant derivatives this fermionic action reads

$$
\begin{gathered}
S^{f}=a^{4} \sum_{x}\left\{\frac { 1 } { 2 a } \sum _ { \mu = 1 } ^ { 4 } \left[\bar{\psi}(x)\left(\gamma_{\mu}+i\left(\gamma_{\mu} \cot \alpha_{\mu}+\gamma_{\mu}^{\prime} \csc \alpha_{\mu}\right)\right) U_{\mu}(x) \psi(x+a \widehat{\mu})\right.\right. \\
\left.-\bar{\psi}(x+a \widehat{\mu})\left(\gamma_{\mu}-i\left(\gamma_{\mu} \cot \alpha_{\mu}+\gamma_{\mu}^{\prime} \csc \alpha_{\mu}\right)\right) U_{\mu}^{\dagger}(x) \psi(x)\right] \\
\left.+\bar{\psi}(x)\left(m_{0}-\frac{i}{a} \sum_{\mu}\left(\gamma_{\mu} \cot \alpha_{\mu}+\gamma_{\mu}^{\prime} \csc \alpha_{\mu}\right)\right) \psi(x)\right\}
\end{gathered}
$$

and it has, like the Wilson action, only nearest-neighbor interactions. This action has the correct continuum limit, and since (as we will see later in Sect. 4) one also has $\left\{\gamma_{\mu}^{\prime}, \gamma_{5}\right\}=0$, it preserves a $U(1)$ chiral symmetry (for $m_{0}=0$ ), which protects from additive mass renormalization, and also satisfies $\gamma_{5}$-hermiticity.

\section{Construction of the generalized action}

We now explain how the generalized action (6) can be derived. We carry out our reasoning in momentum space, and it is sufficient to consider the free case and then at the end insert the gauge interactions. From now on we will set for simplicity $a=1$. 
The standard Boriçi-Creutz action (11) can be viewed as the outcome of an ingenious construction, which was devised by Creutz in [3]. It can be represented as a linear combination of two physically equivalent naive fermion actions (one of them having been given a momentum shift), which are the first and second term in the last line of Eq. (1). The first term vanishes when any component of the momentum is equal to 0 or $\pi$. The zeros of the second term are instead positioned at the momenta $( \pm \pi / 2, \pm \pi / 2, \pm \pi / 2, \pm \pi / 2)$, which are the ones that are maximally distant from the zeros of the first term. So, the 16 zeros corresponding to the doublers of the second naive action are located farthest away from the 16 zeros of the doublers of the first naive action. The particular combination in (1), with the subtraction of $\Gamma$, results in an action with only two zeros.

We first try to use again two naive fermions in order to arrive at a generalized action in which the second zero can be put at an arbitrary location. To do this, it is useful to see the Boriçi-Creutz action written in the following way:

$$
D^{B C}(p)=i \sum_{\mu}\left(\gamma_{\mu} \sin p_{\mu}+\gamma_{\mu}^{\prime} \sin \left(p_{\mu}+\pi / 2\right)\right)-2 i \Gamma+m_{0}
$$

This suggests to make a translation in momentum space of the second naive fermion action, such that its second zero on each of its axes (the one with the negative slope) is moved to a generic position $p_{\mu}=\alpha_{\mu}$ :

$$
D^{B C^{\prime}}(p)=i \sum_{\mu}\left(\gamma_{\mu} \sin p_{\mu}+\gamma_{\mu}^{\prime} \sin \left(p_{\mu}+\pi-\alpha_{\mu}\right)\right)-i \sum_{\mu} \gamma_{\mu}^{\prime} \sin \alpha_{\mu}+m_{0}
$$

Then the second zero of the minimally doubled action (88) is at $p_{\mu}=\alpha_{\mu}$ (the first one still sitting at the origin). Note that also the $\Gamma$ term had to be modified in order to achieve the desired minimal doubling, and is now $\Gamma=(1 / 2) \sum_{\mu} \gamma_{\mu} \sin \alpha_{\mu}=(1 / 2) \sum_{\mu} \gamma_{\mu}^{\prime} \sin \alpha_{\mu}$, and that this action can also be written as

$$
\begin{aligned}
D^{B C^{\prime}}(p) & =i \sum_{\mu}\left(\gamma_{\mu} \sin p_{\mu}+\gamma_{\mu}^{\prime}\left(\sin \left(\alpha_{\mu}-p_{\mu}\right)-\sin \alpha_{\mu}\right)\right)+m_{0} \\
& =i \sum_{\mu}\left(\gamma_{\mu}\left(\sin p_{\mu}-\sin \alpha_{\mu}\right)+\gamma_{\mu}^{\prime} \sin \left(\alpha_{\mu}-p_{\mu}\right)\right)+m_{0} .
\end{aligned}
$$

The mechanism out of which one at the end obtains an action like (8) which has only two zeros is analogous to the one of the standard Boriçi-Creutz action. When the coefficients of both $\gamma_{\mu}$ and $\gamma_{\mu}^{\prime}$ vanish at the same value of the momentum $p_{\mu}$, one has a zero of the action (although this is not the only possibility, because $\gamma_{\mu}$ and $\gamma_{\mu}^{\prime}$ are not independent). This is true for any given spacetime direction and the corresponding momentum component $\mu$. Leaving aside for a moment the last term of the action (8) (the one containing $\sum_{\mu} \gamma_{\mu}^{\prime} \sin \alpha_{\mu}$ ), we can see that the coefficient of $\gamma_{\mu}$ in the first term and that of $\gamma_{\mu}^{\prime}$ in the second term can never vanish together. Moreover, the value of the coefficient of $\gamma_{\mu}^{\prime}$ at the momentum where the coefficient of $\gamma_{\mu}$ vanishes with positive slope, is the same of the coefficient of $\gamma_{\mu}$ at the momentum where the coefficient of $\gamma_{\mu}^{\prime}$ vanishes with negative slope, and is equal to $i \sin \alpha_{\mu}$. That is why one at the end needs to subtract the term with $\sum_{\mu} \gamma_{\mu}^{\prime} \sin \alpha_{\mu}$ from the sum of the two naive actions. The outcome of this construction is that only two zeros are left, one at the origin and the other at $\alpha_{\mu}$. 
There is however a major problem with the action (8), and it is that it does not have the correct continuum limit. Indeed, its leading terms for small $p$ are

$$
D^{B C^{\prime}}(p) \simeq i \not p-i \sum_{\mu} \gamma_{\mu}^{\prime} p_{\mu} \cos \alpha_{\mu}
$$

and one consequence of this is that the basic vertex for the emission of a gluon by a quark current is not simply proportional to $\gamma_{\mu}$, but contains also $\gamma_{\mu}^{\prime}$ terms, even in the continuum limit. Then this action is not suitable to be used in Monte Carlo simulations.

The wrong continuum limit originates from the fact that at the point $p_{\mu}=(0,0,0,0)$, where the coefficient of $i \gamma_{\mu}$ vanishes, the first derivative of the function expressing the coefficient of $i \gamma_{\mu}^{\prime}$ does not vanish. One must then find a way to overcome this limitation.

In order to obtain that this derivative becomes zero, we have to modify in a suitable way the shape of the naive actions in momentum space. This can be accomplished by making the substitution

$$
\sin p_{\mu} \longrightarrow \sin p_{\mu}-\cot \alpha_{\mu}\left(1-\cos p_{\mu}\right)
$$

This new function is now taken as the coefficient of $i \gamma_{\mu}$. It is easy to see that on each $\mu$ axis it has zeros at $p_{\mu}=0$ and $p_{\mu}=2 \alpha_{\mu}$, with slopes 1 and -1 respectively (as it should be). Its maximum is attained at $p_{\mu}=\alpha_{\mu}$, with value $\left(1-\cos \alpha_{\mu}\right) / \sin \alpha_{\mu}$, and its minimum at $p_{\mu}=\alpha_{\mu}-\pi$, with value $-\left(1+\cos \alpha_{\mu}\right) / \sin \alpha_{\mu}$. The coefficient of $i \gamma_{\mu}^{\prime}$ is given by a function in momentum space of the same shape as (10), but on which a negative shift of length $\alpha_{\mu}$ has been applied. Its zeros are then located at $-\alpha_{\mu}$ and $\alpha_{\mu}$, and the position of the maximum is also shifted accordingly.

The mechanism of minimal doubling is now similar to the one sketched above. The main difference is that at $p_{\mu}=0$, where the coefficient of $\gamma_{\mu}$ is zero, that of $\gamma_{\mu}^{\prime}$ has now a maximum, and thus its first derivative is zero. At $p_{\mu}=\alpha_{\mu}$ the roles of $\gamma_{\mu}$ and $\gamma_{\mu}^{\prime}$ are simply reversed. The (free) minimally doubled Dirac operator coming out of this choice of modified naive actions is then

$$
\begin{aligned}
D(p)=i \sum_{\mu}\left[\gamma_{\mu}\left(\sin p_{\mu}-\cot \alpha_{\mu}\left(1-\cos p_{\mu}\right)\right)\right. \\
\left.+\gamma_{\mu}^{\prime}\left(\sin \left(p_{\mu}+\alpha_{\mu}\right)-\cot \alpha_{\mu}\left(1-\cos \left(p_{\mu}+\alpha_{\mu}\right)\right)\right)\right]-i n \Gamma+m_{0} \\
=i \sum_{\mu} \frac{1}{\sin \alpha_{\mu}}\left[\gamma_{\mu}\left(\cos \left(p_{\mu}-\alpha_{\mu}\right)-\cos \alpha_{\mu}\right)\right. \\
\left.+\gamma_{\mu}^{\prime}\left(\cos p_{\mu}-\cos \alpha_{\mu}\right)\right]-i n \Gamma+m_{0},
\end{aligned}
$$

where a new definition of $\Gamma$ must be now used, as explained in the next Section. After Fourier transforming to position space and then adding the gauge interactions, this action corresponds to the expression given in Eq. (6)

One can check that the continuum limit is now the correct one, and indeed the leading term for small $p$ of (11) is $i \not p$, as it should be. What has happened is that $\gamma_{\mu}^{\prime}$ terms like the ones in Eq. (9), which spoiled the continuum limit for the action (8), are now exactly compensated by the new $\cot \alpha_{\mu}$ terms that have been introduced in the modified naive actions. 


\section{Dirac matrices}

The generalized definition of $\Gamma$ that, when combined with the sum of the modified naive actions, builds a minimally doubled action is

$$
\Gamma=\frac{1}{n} \sum_{\mu} \frac{1-\cos \alpha_{\mu}}{\sin \alpha_{\mu}} \gamma_{\mu}=\frac{1}{n} \sum_{\mu} \frac{1-\cos \alpha_{\mu}}{\sin \alpha_{\mu}} \gamma_{\mu}^{\prime} ; \quad n=\sqrt{\sum_{\mu} \frac{\left(1-\cos \alpha_{\mu}\right)^{2}}{\sin ^{2} \alpha_{\mu}}} .
$$

With this choice the action (11) has always two zeros, located at the origin and at $\alpha_{\mu}$. If the components of $\alpha_{\mu}$ become large, other zeros can however appear, as discussed in the next Section.

The matrix $\Gamma$ encodes the generic direction of hypercubic breaking that is now possible to choose. Note that one can also write it as

$$
\Gamma=\frac{1}{n} \sum_{\mu} \gamma_{\mu} \tan \left(\alpha_{\mu} / 2\right)
$$

from which it is perhaps easier to see that there is a one-to-one correspondence between $\Gamma$ and the direction of hypercubic breaking.

The normalization of the hermitian matrix $\Gamma$ in (12) is such that $\Gamma^{2}=1$, and so this matrix is also unitary. We can then observe that also the two modified naive actions out of which the action (11) was built are physically equivalent. Indeed if, along the lines of [3], we consider the unitary transformation

$$
\begin{aligned}
& \psi(x) \rightarrow e^{-i \alpha_{\mu} x_{\mu}} \Gamma \psi(x) \\
& \bar{\psi}(x) \rightarrow e^{i \alpha_{\mu} x_{\mu}} \bar{\psi}(x) \Gamma
\end{aligned}
$$

in momentum space the corresponding effect is given by the substitutions $\sin \left(p_{\mu}\right) \rightarrow$ $\sin \left(p_{\mu}+\alpha_{\mu}\right)$ and $\cos \left(p_{\mu}\right) \rightarrow \cos \left(p_{\mu}+\alpha_{\mu}\right)$. Thus, under this unitary transformation the first modified naive action goes exactly into the second one.

An important consequence of this equivalence is that the relation $\gamma_{\mu}^{\prime}=\Gamma \gamma_{\mu} \Gamma$ of the standard Boriçi-Creutz action is still valid, even though now the explicit expressions of the $\gamma_{\mu}^{\prime}$ matrices depend on the choice of $\alpha_{\mu}$. 3 Moreover, from this relation (and together with $\Gamma^{2}=1$ ) the equivalence of the two definitions of $\Gamma$ in Eq. (12) can be verified, as well as that

$$
\left\{\gamma_{\mu}^{\prime}, \gamma_{\nu}^{\prime}\right\}=\left\{\Gamma \gamma_{\mu} \Gamma, \Gamma \gamma_{\nu} \Gamma\right\}=\Gamma\left\{\gamma_{\mu}, \gamma_{\nu}\right\} \Gamma=2 \delta_{\mu \nu}
$$

which indicates that the matrices $\gamma_{\mu}^{\prime}$ constitute a fully legitimate set of Dirac matrices. In general they are a linear combination of the $\gamma_{\mu}$, which can be expressed as $\gamma_{\mu}^{\prime}=$ $\sum_{\nu} a_{\mu \nu} \gamma_{\nu}$, where $a$ is an orthogonal matrix [2]. The specific values of the entries of $\gamma_{\mu}^{\prime}$ depend on the actual location of the second zero.

It is also easy to see, using $\gamma_{\mu}^{\prime}=\Gamma \gamma_{\mu} \Gamma$, that $\left\{\gamma_{\mu}^{\prime}, \gamma_{5}\right\}=0$, and from this the chiral symmetry and the $\gamma_{5}$-hermiticity of the action immediately follow. Another useful

\footnotetext{
${ }^{3}$ This equivalence can be seen from the fact that the unitary transformation brings the first zero onto the second one, and so $\bar{\psi} \gamma_{\mu} \psi \rightarrow \bar{\psi} \Gamma \gamma_{\mu} \Gamma \psi=\bar{\psi} \gamma_{\mu}^{\prime} \psi$.
} 
relation for the $\gamma_{\mu}^{\prime}$ matrices is 4

$$
\gamma_{\mu}^{\prime}=\left\{\Gamma, \gamma_{\mu}\right\} \Gamma-\gamma_{\mu}=\frac{2}{n} \frac{1-\cos \alpha_{\mu}}{\sin \alpha_{\mu}} \Gamma-\gamma_{\mu}
$$

Note that it must be $\alpha_{\mu} \neq 0$ and $\alpha_{\mu} \neq \pi$, otherwise the two modified naive actions of Sect. 3 collapse onto each other or their sum is identically zero, and thus the construction of the action (11) obviously degenerates. The direction of hypercubic breaking can then never exactly correspond to one of the $p_{\mu}$ axes. Note also that writing $\Gamma=(1 / n) \sum_{\mu} \gamma_{\mu} \tan \left(\alpha_{\mu} / 2\right)$ could erroneously suggest that $\alpha_{\mu}=0$ might also be included, however this cannot be a legitimate choice.

If the second zero is constrained to remain on the positive major diagonal, i.e. $\alpha_{\mu}=(\alpha, \alpha, \alpha, \alpha)$, then the formula for $\Gamma$ reduces to the simple expression of Eq. (3), the one of the standard Boriçi-Creutz action. This also happens with other relations like the one in (17).

For $\alpha_{\mu}=(\pi / 2, \pi / 2, \pi / 2, \pi / 2)$ all above formulae reduce to those of the standard Boriçi-Creutz action. In particular, the normalization factor can be seen to behave like $n \rightarrow 2$ when $\alpha_{\mu} \rightarrow \pi / 2$.

What we have generalized here is the standard Boriçi-Creutz action whose second zero is conventionally taken at $(\pi / 2, \pi / 2, \pi / 2, \pi / 2)$, and hence its direction of hypercubic breaking is the positive major diagonal. However, from the second (modified) naive action one could choose any of its other 15 zeros out of $( \pm \pi / 2, \pm \pi / 2, \pm \pi / 2, \pm \pi / 2)$ to survive at the end in the final combination (11). If for instance one picks $(\pi / 2,-\pi / 2, \pi / 2, \pi / 2)$ as the second zero, then the new direction of hypercubic breaking is a different major hypercubic diagonal, and reflected in the new choice $\Gamma=\frac{1}{2}\left(\gamma_{1}-\gamma_{2}+\gamma_{3}+\gamma_{4}\right)$. Each of these 16 possible choices corresponds to a restriction to a four-dimensional orthant, that is to only one sixteenth of the whole first Brillouin zone. The generalized action (11) that we have derived is instead valid for all the sixteen orthants combined (except for the $p_{\mu}$ axes). One can see that also the expression for $\Gamma$ given in (12) already covers this general case, and for example if $-\pi<\alpha_{2}<0$ then the coefficient of $\gamma_{2}$ in (12) becomes automatically negative.

It is thus in general possible to choose $\alpha_{\mu}$ in any location as second zero of the action, as long as every component of it differs from 0 or $\pi$.

However, not all possible choices of $\alpha_{\mu}$ in the first Brillouin zone preserve minimal doubling. As we discuss in the next Section, in general additional zeros can appear if some components of $\alpha_{\mu}$ become too large. It can however be proven that for a large region of choices of $\alpha_{\mu}$ there are indeed only two flavors.

\section{Minimal doubling}

It is interesting to note that a special situation for the modified naive actions arises when any component of $\alpha_{\mu}$ becomes exactly $\alpha_{\mu}=2 \pi / 3$. Since in this case (and only in

\footnotetext{
${ }^{4}$ This relation was $\gamma_{\mu}^{\prime}=\Gamma-\gamma_{\mu}$ for the standard Boriçi-Creutz action. The relation $\gamma_{\mu}^{\prime}=\Gamma \gamma_{\mu} \Gamma$ remains instead unmodified also in the generalized Boriçi-Creutz action, and it so looks as though it could be the more fundamental of the two main ways of expressing $\gamma_{\mu}^{\prime}$ in terms of $\gamma_{\mu}$.
} 
this case, up to sign flips of the components) $\alpha_{\mu}$ satisfies $2 \alpha_{\mu}-2 \pi=-\alpha_{\mu}$, what happens is that the $p_{\mu}$ component of one zero of the first modified naive action coincides with the $p_{\mu}$ component of one zero of the second modified naive action. This is the only case for which this can happen (together with its mirror case $\alpha_{\mu}=-2 \pi / 3$ ).

It actually turns out that for $\alpha_{\mu}=(2 \pi / 3,2 \pi / 3,2 \pi / 3,2 \pi / 3)$ the generalized BoriçiCreutz action has indeed additional zeros, such as 5

$$
p_{\mu}=(\pi / 3, \pi / 3, \pi / 3,-2 \pi / 3)
$$

This is not entirely trivial, as for these particular values of $\alpha_{\mu}$ and $p_{\mu}$ the action is at first sight given by

$$
D(p)=i \frac{2}{\sqrt{3}} \sum_{k=1}^{3}\left(\gamma_{k}+\gamma_{k}^{\prime}\right)-i n \Gamma,
$$

with $n=2 \sqrt{3}$. However, if one now uses $\gamma_{k}^{\prime}=(2 \sqrt{3} / n) \Gamma-\gamma_{k}$ from Eq. (17), then all terms cancel out (even without expanding $\Gamma$ ), and so $D(p)$ indeed vanishes. As noted before, all nontrivial permutations of the components of this $p_{\mu}$ also correspond to additional zeros of the action.6

Any zero of the action (11) has to satisfy the trace equations

$$
\sum_{\mu} \frac{\cos \left(p_{\mu}-\alpha_{\mu} / 2\right)}{\cos \left(\alpha_{\mu} / 2\right)}=4, \quad \frac{\sin \left(p_{\mu}-\alpha_{\mu} / 2\right)}{\sin \left(\alpha_{\mu} / 2\right)}=\frac{\sin \left(p_{\nu}-\alpha_{\nu} / 2\right)}{\sin \left(\alpha_{\nu} / 2\right)}
$$

which are introduced and discussed in more detail in Appendix $\mathrm{A}$, and come out from imposing $\operatorname{Tr}\left(\gamma_{\mu} \sin \alpha_{\mu} /\left(1-\cos \alpha_{\mu}\right)-\gamma_{\nu} \sin \alpha_{\nu} /\left(1-\cos \alpha_{\mu}\right)\right) D(p)=0$ and $\operatorname{Tr} \Gamma D(p)=0$ respectively. It is easy to verify that the values of $\alpha_{\mu}$ and $p_{\mu}$ given above for the additional zero satisfy these trace equations.

Two important general properties of the zeros can be straightforwardly inferred from the trace equations.

\footnotetext{
${ }^{5}$ I thank Mike Creutz for alerting me to the existence of this additional zero.

${ }^{6}$ It might be interesting to observe that also the action with the wrong continuum limit, Eq. (8), becomes additional zeros for the same value of $\alpha=2 \pi / 3$, and at the same locations in momentum space, $p_{\mu}=(\pi / 3, \pi / 3, \pi / 3,-2 \pi / 3)$ plus its nontrivial permutations. The trace equations for this action, analogous to those discussed in Appendix $\AA$ are $\operatorname{Tr}\left(\gamma_{\mu} / \sin \alpha_{\mu}-\gamma_{\nu} / \sin \alpha_{\nu}\right) D(p)=0$ and $\operatorname{Tr} \Gamma D(p)=0$, and give respectively

$$
\frac{\sin p_{\mu}-\sin \left(\alpha_{\mu}-p_{\mu}\right)}{\sin \alpha_{\mu}}=\frac{\sin p_{\nu}-\sin \left(\alpha_{\nu}-p_{\nu}\right)}{\sin \alpha_{\nu}}
$$

and

$$
\sum_{\mu} \sin \alpha_{\mu}\left(\sin p_{\mu}-\sin \alpha_{\mu}+\sin \left(\alpha_{\mu}-p_{\mu}\right)\right)=0 .
$$

It can be easily verified that the values of $\alpha_{\mu}$ and $p_{\mu}$ given above satisfy these trace equations (in addition to the "standard" zeros). This happens in spite of the fact that for the naive (not modified) actions of Eq. (8) this value of $\alpha_{\mu}$ (and indeed any other one) does not give rise to a special situation. It could be that this particular additional zero at this specific location is generated by some general underlying mechanism, to a certain extent independent of the details of this kind of minimally doubled actions. Whether this is indeed a general feature of all these actions remains still to be understood.
} 
One is that there is a complete symmetry of the zeros under permutations of the coordinates. Hence, in the following all zeros will be meant and given up to nontrivial permutations of $\alpha_{\mu}$ and $p_{\mu}$.

The other one follows from the fact that these equations are symmetric under reflections of any of the coordinates axes. Then, if a certain $p_{\mu}$ is a zero for the action given by $\alpha_{\mu}$, changing sign to one component of $p_{\mu}$ will automatically give a zero of the action corresponding to an $\alpha_{\mu}$ which has also undergone the same change of sign. An important consequence of this is that each orthant can then be studied separately, since the distribution patterns of the zeros is the same in every orthant, and only changes of signs have to be taken into account. We can then restrict our considerations to $\alpha_{\mu}$ 's which have only positive components, that is to the first orthant (which corresponds to generalizing, of the 15 standard Boriçi-Creutz actions, the one where the second zero is on the positive major diagonal). In the following discussions $0<\alpha_{\mu}<\pi$ will be always understood.

With the help of these trace equations one can always check, by direct inspection, whether or not a given $p_{\mu}$ is a zero of the action for a given choice of $\alpha_{\mu}$. It is easy to see that these equations are satisfied by the two "standard" zeros $p_{\mu}=(0,0,0,0)$ and $p_{\mu}=\left(\alpha_{\mu}, \alpha_{\mu}, \alpha_{\mu}, \alpha_{\mu}\right)$, and that mixed choices such as $p_{\mu}=\left(\alpha_{1}, 0, \alpha_{3}, \alpha_{4}\right)$ instead are not zeros of the action.

Thus, the generalized action has always two zeros at the locations $p_{\mu}=(0,0,0,0)$ and $p_{\mu}=\left(\alpha_{\mu}, \alpha_{\mu}, \alpha_{\mu}, \alpha_{\mu}\right)$. For certain regions in the space of $\alpha_{\mu}$ however additional zeros can arise, leading so to the loss of minimal doubling.

We start to look at the situation where $\alpha_{\mu}$ lies on the positive major diagonal, i.e. $\alpha_{\mu}=(\alpha, \alpha, \alpha, \alpha)$. Then the trace equations for the zeros become much simpler, and one can solve them analytically along the entire length of the diagonal, and in this way see where there are extra zeros and give an explicit expression for their locations. The detailed derivations of the following results can be found in Appendix A.

One thing that can be easily proven is that with this restriction on $\alpha_{\mu}$ no extra zero of the form $p_{\mu}=(p, p, p, p)$ can exist, because any zero of this form satisfies the simple equation

$$
D(p)=\cos (p-\alpha)+\cos (p)-\cos (\alpha)-1=0,
$$

which has no additional solutions besides the "standard" ones given by $p=0$ and $p=\alpha$. So, wherever there are additional doublers on this diagonal of $\alpha_{\mu}$, these cannot have all components of $p_{\mu}$ equal.

For $\alpha_{\mu}=(\alpha, \alpha, \alpha, \alpha)$ one can prove (see Appendix $\mathrm{A}$ ) that if one chooses $\alpha<2 \pi / 3$ there cannot be additional zeros, and thus minimal doubling is preserved. On the other hand, when $\alpha \geq 2 \pi / 3$ additional doublers do appear, and they can be expressed (as solution of Eqs. (45)) as

$$
p_{\mu}=\left(\alpha / 2+\eta_{+}, \alpha / 2+\eta_{+}, \alpha / 2+\eta_{+}, \alpha / 2+\eta_{-}\right), \quad \eta_{ \pm}=\arccos ( \pm 2 \cos \alpha / 2) .
$$

This gives 2 solutions for each choice of $\alpha$ (up to nontrivial permutations of the components), which become more and more distant from each other as the value of $\cos \alpha / 2$ decreases towards 0 , where they coalesce on the standard zeros. At the other end of the range, i.e. for $\alpha=2 \pi / 3$, the two solutions become degenerate, and correspond to the additional zero (18). 
In the general case where $\alpha_{\mu}$ is not on a major hypercubic diagonal it is difficult to obtain exact solutions to the trace equations. However one can prove that minimal doubling can be guaranteed if the components of $\alpha_{\mu}$ do not become too large. A uniform bound for all components is provided by

$$
\cos \left(\alpha_{\mu} / 2\right) \geq \frac{3}{5}
$$

which corresponds to $\alpha_{\mu} \leq 0.590334 \pi \sim 106.26^{\circ}$. When this condition is true no other zeros can appear in the action. Its derivation is given in Appendix A.

One can also see, by direct inspection of the trace equations, that there are extra zeros for the actions defined by ${ }^{7}$

$$
\cos \left(\alpha_{\mu} / 2\right)=\left(\frac{3-3 \delta}{5-4 \delta}, \frac{3-3 \delta}{5-4 \delta}, \frac{3-3 \delta}{5-4 \delta}, 1-\delta\right),
$$

and these zeros are given by

$$
\cos \left(p_{\mu}-\alpha_{\mu} / 2\right)=(1,1,1,-1)
$$

If one takes $\delta$ to be very small, the existence of these zeros shows that it is not possible to further improve the uniform bound (25) given above.

It can also be demonstrated that when for all components

$$
\cos \left(\alpha_{\mu} / 2\right) \leq \frac{1}{2}
$$

then minimal doubling is surely lost, that is extra zeros always appear and one is in another branch. The derivation of this statement can also be found in Appendix $\mathrm{A}$, and this region corresponds to taking $\alpha_{\mu} \geq 2 \pi / 3$.

Somewhere inbetween the two uniform bounds established above, $\cos \left(\alpha_{\mu} / 2\right) \geq 3 / 5$ and $\cos \left(\alpha_{\mu} / 2\right) \leq 1 / 2$, there should be a 3 -dimensional surface of demarcation between the domain of minimal doubling and the branches which contain more doublers. Note that the extra zeros just given above lie infinitesimally close to the bound for the minimal doubling region, and that conversely (as found at the beginning of this Section) when one moves on the major diagonal towards the origin starting from the additional zero for the action $\alpha_{\mu}=(2 \pi / 3,2 \pi / 3,2 \pi / 3,2 \pi / 3)$, which lies exactly at the bound $\cos \left(\alpha_{\mu} / 2\right)=1 / 2$, minimal doubling is immediately restored. It also seems from these results that the region of minimally doubling contracts in the directions where the differences between the components of $\alpha_{\mu}$ are large, whereas when all components of $\alpha_{\mu}$ are equal then minimal doubling can be preserved also for larger values of them. It is possible that the boundary of the minimal doubling domain will have a nontrivial and complicated shape.

It is not easy to derive a general solution of the trace equations when $\alpha_{\mu}$ is located outside a major hypercubic diagonal. However it is possible to decouple them and so

\footnotetext{
${ }^{7}$ Note that it must be $\delta>0$, and that we cannot take here $\delta=0$, because this corresponds to $\alpha_{4}=0$, in which case, as remarked in the previous Section, the construction of the action (11) degenerates. Note also that for $\delta=1 / 2$ one obtains again the additional zero (18).
} 
to write an equation for a single component of $p_{\mu}$, which could turn out to be useful in other contexts. Indeed, by combining Eq. (39) with Eq. (37) one can obtain an equation for one component $\cos \left(p_{\sigma}-\alpha_{\sigma} / 2\right)$,

$$
\frac{\cos \left(p_{\sigma}-\alpha_{\sigma} / 2\right)}{\cos \left(\alpha_{\sigma} / 2\right)}+\sum_{\rho \neq \sigma}( \pm) \frac{1}{\cos \left(\alpha_{\rho} / 2\right)} \sqrt{1-\left(1-\cos ^{2}\left(\alpha_{\rho} / 2\right)\right) \frac{1-\cos ^{2}\left(p_{\sigma}-\alpha_{\sigma} / 2\right)}{1-\cos ^{2}\left(\alpha_{\sigma} / 2\right)}}=4
$$

where \pm means that for each $\rho$ component one can take either the positive or the negative square root. Any component $\mu$ of a momentum $p_{\mu}$ which is a zero of the action must then satisfy one of these 9 possible equations.

One can further explore the whole space spanned by $\alpha_{\mu}$, even though at this stage we have already a general picture of the situation which is sufficient for first nonperturbative investigations. After all, the values of $\alpha_{\mu}$ for which $\cos \left(\alpha_{\mu} / 2\right)=3 / 5$ and $\cos \left(\alpha_{\mu} / 2\right)=1 / 2$ do not lie too distant from each other. Of course, if needed one can always immediately check from the trace equations, by direct inspection, whether a certain momentum $p_{\mu}$ is a zero of the action or not.

Moreover, one should keep in mind that the numbers that we have given in this Section are the result of tree-level considerations, and hence the actual surfaces of demarcation between the regions of minimal doubling and those that contain additional doublers may be slightly different after all interactions have been taken into account.

\section{Outlook}

In this article we have presented new minimally doubled actions of the Boriçi-Creutz type, with which one is able to put the second zero at any location in momentum space (except on the $p_{\mu}$ axes). Minimal doubling is preserved provided the components of $\alpha_{\mu}$ do not become too large. We have indeed proven that there are always only two zeros in the first orthant when for all components $\cos \left(\alpha_{\mu} / 2\right) \geq 3 / 5$, while if for all components $\cos \left(\alpha_{\mu} / 2\right) \leq 1 / 2$ then surely there are at least four zeros. Exact solutions can be given over the whole length of the positive major diagonal, and in this case minimal doubling is preserved also for larger values of the components of $\alpha_{\mu}$, that is when $\cos \left(\alpha_{\mu} / 2\right)>1 / 2$. This pattern is exactly repeated in the 15 other orthants.

Recently new minimally doubled actions of a still different kind [15] were also proposed, which allow to put the second zero anywhere on one of the $p_{\mu}$ axes. Thus, with all these new actions at hand, one can now choose to put the second zero at any position in a region which covers a large part of the first Brillouin zone.

It seems not possible to go continuously from one kind of actions to the other one. For example, if one takes $\alpha_{\mu}=(\epsilon, \epsilon, \epsilon, \pi-\epsilon)$ in the generalized Boriçi-Creutz action (11), and then lets $\epsilon \rightarrow 0$, a singularity of the action is encountered, and one cannot connect smoothly to the standard Karsten-Wilczek action. Even taking just

$\alpha_{\mu}=(\epsilon, \epsilon, \epsilon,-2 \alpha)$ and letting $\epsilon \rightarrow 0$ while $\alpha$ is kept constant, produces a singularity of the action before the position of the second zero of the generalized Karsten-Wilczek actions can be reached. This should perhaps be not too surprising, as the mechanism out of which one can remain with only two zeros is substantially different for these two 
kinds of actions. We also note that for these generalized Boriçi-Creutz fermions the (4dimensional) distance can be increased up to $4 \pi / 3$ (on the major hypercubic diagonals) while still preserving minimal doubling, and this is larger than the maximum distance possible with (even generalized) Karsten-Wilczek fermions.

There is also another difference, if we look at the bare actions. For KarstenWilczek fermions, the generalized case [15] included, $P$ is a conserved symmetry, but $T$ and $C$ are violated [16], whereas for standard Boriçi-Creutz fermions as well as their generalization presented here, also $P$ is violated. A violation of $C$, which occurs for all these bare actions, has among others the consequence that the masses of the $\pi^{+}$and $\pi^{-}$are not equal. This seems hard to escape, because PT can never be a symmetry of any minimally doubled action, as was explained in [16]. Of course $C$ can be restored at the end and consequently the masses of the $\pi^{+}$and $\pi^{-}$become again equal, once one has properly tuned the required counterterms and thus constructed the properly renormalized action to be eventually used in Monte Carlo simulations.

For Karsten-Wilczek fermions the counterterms contain factors like $\delta_{\mu 4}$. For standard Boriçi-Creutz fermions the characteristic feature of the counterterms is the appearance of sums involving only one Lorentz index, $\sum_{\mu} f_{\mu}$, which mirrors $2 \Gamma=\sum_{\mu} \gamma_{\mu}$ [11. In the case of the generalized Boriçi-Creutz fermions presented here, the sums over only one Lorentz index must be of the form $\sum_{\mu} f_{\mu}\left(1-\cos \alpha_{\mu}\right) / \sin \alpha_{\mu}$, which mirrors the generalized $\Gamma$. We expect then the fermionic counterterms to look formally like the ones required for standard Boriçi-Creutz fermions,

$$
\bar{\psi} \Gamma \sum_{\mu} D_{\mu} \psi, \quad \frac{1}{a} \bar{\psi}(x) \Gamma \psi(x),
$$

where of course the explicit expressions now depend on the actual choice of $\alpha_{\mu}$. The gluonic counterterm will also contain information about the special direction, and we expect it to be a fixed linear combination of the components of the plaquette which reflects the specific direction of hypercubic breaking, with only one overall coefficient to be tuned:

$$
\sum_{\mu \nu \rho} \frac{1-\cos \alpha_{\mu}}{\sin \alpha_{\mu}} \frac{1-\cos \alpha_{\nu}}{\sin \alpha_{\nu}} \operatorname{Tr} F_{\mu \rho}(x) F_{\rho \nu}(x) .
$$

Note that a further splitting of the index $\rho$ into two independent sums gives identically zero, because of the antisymmetry of the field-strength tensor.

It is possible that special choices of $\alpha_{\mu}$ can result in a reduction of the number of counterterms, as it has occurred in the case of generalized Karsten-Wilczek fermions [15]. One should investigate the renormalization properties of this action and consequently pick up the best choice of $\alpha_{\mu}$ for Monte Carlo simulations. The hope is that for some special value of $\alpha_{\mu}$ the corresponding action would require no counterterms at all.

It appears difficult to insert more parameters in these actions of the Boriçi-Creutz type, beyond $\alpha_{\mu}$. A parameter like the $\lambda$ of the generalized Karsten-Wilczek case cannot be introduced, as this is very peculiar to that kind of minimally doubled fermions, in which the mechanism of minimal doubling is different from the one of the Boriçi-Creutz action. But even with $\alpha_{\mu}$ alone, we can vary at will not only the distance between the two zeros, but also the direction of hypercubic breaking. The four components of $\alpha_{\mu}$ can 
be chosen independently, and it could be that one can regard $\alpha_{\mu}$ as four independent parameters (in this sense, many more than for the generalized Karsten-Wilczek fermions studied in [15]). If the four components of $\alpha_{\mu}$ really behave completely independently under renormalization, this could increase the possibility for finding some particular choice of parameters which realizes a minimally doubled action with no counterterms.

\section{Acknowledgments}

I have enjoyed and profited from many interesting conversations with Mike Creutz, whom I warmly thank.

\section{A Derivations of minimal doubling}

In this Appendix we provide some derivations regarding the properties of the zeros of the action and the possible appearance in specific domains of extra doublers which destroy minimal doubling.

\section{A.1 Trace equations}

We need the basic traces

$$
\operatorname{Tr} \Gamma \gamma_{\mu}=\operatorname{Tr} \Gamma \gamma_{\mu}^{\prime}=\frac{4}{n} \frac{1-\cos \alpha_{\mu}}{\sin \alpha_{\mu}}
$$

and

$$
\operatorname{Tr} \gamma_{\mu} \gamma_{\nu}^{\prime}=-4 \delta_{\mu \nu}+\frac{8}{n^{2}} \frac{1-\cos \alpha_{\mu}}{\sin \alpha_{\mu}} \frac{1-\cos \alpha_{\nu}}{\sin \alpha_{\nu}}
$$

We start by computing

$$
\begin{aligned}
\operatorname{Tr} \gamma_{\mu} D(p)=4 i\{ & \sin p_{\mu}+\cos p_{\mu} \frac{\cos \alpha_{\mu}-1}{\sin \alpha_{\mu}} \\
& \left.+\frac{1-\cos \alpha_{\mu}}{\sin \alpha_{\mu}}\left(\frac{2}{n^{2}} \sum_{\rho} \frac{1-\cos \alpha_{\rho}}{\sin ^{2} \alpha_{\rho}}\left(\cos p_{\rho}-\cos \alpha_{\rho}\right)-1\right)\right\},
\end{aligned}
$$

and also the computation of $\operatorname{Tr} \gamma_{\mu}^{\prime} D(p)$ gives a similar result of this kind. Then one can see that it is more useful to consider

$$
\operatorname{Tr}\left(\frac{\sin \alpha_{\mu}}{1-\cos \alpha_{\mu}} \gamma_{\mu}-\frac{\sin \alpha_{\nu}}{1-\cos \alpha_{\nu}} \gamma_{\nu}\right) D(p)=-\operatorname{Tr}\left(\frac{\sin \alpha_{\mu}}{1-\cos \alpha_{\mu}} \gamma_{\mu}^{\prime}-\frac{\sin \alpha_{\nu}}{1-\cos \alpha_{\nu}} \gamma_{\nu}^{\prime}\right) D(p) \text {. }
$$

Then, from (34) we can easily obtain the simple result

$$
\operatorname{Tr}\left(\frac{\sin \alpha_{\mu}}{1-\cos \alpha_{\mu}} \gamma_{\mu}-\frac{\sin \alpha_{\nu}}{1-\cos \alpha_{\nu}} \gamma_{\nu}\right) D(p)=4 i\left(\frac{\sin \left(p_{\mu}-\alpha_{\mu} / 2\right)}{\sin \left(\alpha_{\mu} / 2\right)}-\frac{\sin \left(p_{\nu}-\alpha_{\nu} / 2\right)}{\sin \left(\alpha_{\nu} / 2\right)}\right) .
$$

If $p$ is a zero of the action, then these traces must also vanish, and the above result implies

$$
\frac{\sin \left(p_{\mu}-\alpha_{\mu} / 2\right)}{\sin \left(\alpha_{\mu} / 2\right)}=\frac{\sin \left(p_{\nu}-\alpha_{\nu} / 2\right)}{\sin \left(\alpha_{\nu} / 2\right)}
$$


These equalities (valid for all directions) are obviously satisfied by the two zeros of the action, $p_{\mu}=0$ and $p_{\mu}=\alpha_{\mu}$. They also prohibit mixed choices like $p_{\mu}=\left(\alpha_{1}, 0, \alpha_{3}, \alpha_{4}\right)$ to be a zero.

We next compute

$$
\operatorname{Tr} \Gamma D(p)=\frac{4 i}{n} \sum_{\mu} \frac{1-\cos \alpha_{\mu}}{\sin \alpha_{\mu}}\left(\sin p_{\mu}+\cos p_{\mu} \frac{\cos \alpha_{\mu}+1}{\sin \alpha_{\mu}}-2 \frac{\cos \alpha_{\mu}}{\sin \alpha_{\mu}}\right)-4 i n .
$$

Equating to zero this result we arrive, after some rearrangements, to

$$
\sum_{\mu} \frac{\cos \left(p_{\mu}-\alpha_{\mu} / 2\right)}{\cos \left(\alpha_{\mu} / 2\right)}=4
$$

This is again satisfied by the two standard zeros of the action, and for them all four terms in the sum are equal to one.

As we already noted in Sect. 5, from the trace equations one can clearly see that the zeros are symmetric under permutations of the coordinates and under reflections of any of the coordinates axes. In particular, this allows us to restrict all the following discussions to the first orthant, that is $0<\alpha_{\mu} / 2<\pi / 2$, which also implies that both $\sin \left(\alpha_{\mu} / 2\right)$ and $\cos \left(\alpha_{\mu} / 2\right)$ are always positive.

\section{A.2 On a major diagonal}

In the particular case in which $\alpha_{\mu}$ lies on a major hypercubic diagonal, the trace equations become much simpler and they can be solved for the entire length of the diagonal. Indeed, when $\alpha_{\mu}=(\alpha, \alpha, \alpha, \alpha)$ Eq. (37) becomes

$$
\sin \left(p_{\mu}-\alpha / 2\right)=\sin \left(p_{\nu}-\alpha / 2\right)
$$

which implies

$$
\cos \left(p_{\mu}-\alpha / 2\right)= \pm \cos \left(p_{\nu}-\alpha / 2\right)
$$

and Eq. (39) becomes

$$
\sum_{\mu} \cos \left(p_{\mu}-\alpha / 2\right)=4 \cos (\alpha / 2)
$$

In the first orthant it is always $\alpha>0$. Now, all cosines on the left hand side of the last equation must have, because of Eq. (41), the same absolute value. Then either they are all of the same sign (in this case, positive), or one of them has opposite sign (in this case, negative) with respect to the other three. The solutions of the first case give the standard zeros, and those of the second case (if they exist) the additional doublers.

It is easy to prove that the second possibility can be excluded if $\alpha<2 \pi / 3$, and thus for this domain of $\alpha$ one maintains minimal doubling. Indeed, Eq. (42) with the constraint $\cos \alpha / 2>1 / 2$ means that

$$
\sum_{\mu} \cos \left(p_{\mu}-\alpha / 2\right)>2
$$


and this implies that, since all $\cos \left(p_{\mu}-\alpha / 2\right)$ must have the same absolute value, then none of them can be negative, because otherwise the result of the sum would be smaller than 2. Thus, all cosines must be equal and have the same sign, and Eq. (42) becomes

$$
\cos \left(p_{\mu}-\alpha / 2\right)=\cos \alpha / 2
$$

which can only be satisfied by the two "standard" zeros $p_{\mu}=(0,0,0,0)$ and $p_{\mu}=$ $(\alpha, \alpha, \alpha, \alpha)$. It is also easy to see that mixed choices such as $p_{\mu}=(\alpha, 0, \alpha, \alpha)$ cannot be zeros of the action, because they do not satisfy Eq. (40).

If, on the other hand, we are in the region $\cos \alpha / 2 \leq 1 / 2$, then Eq. (42) can still have solutions for $p_{\mu}$ even when one of the cosines in its left hand side is negative, such as

$$
\cos \left(p_{\mu}-\alpha / 2\right)=(2 \cos \alpha / 2,2 \cos \alpha / 2,2 \cos \alpha / 2,-2 \cos \alpha / 2) .
$$

These are then extra zeros. It is easy to see that for $\alpha=2 \pi / 3$ there is only one solution, the one that we have already discussed in Sect. 5, $p_{\mu}=(\pi / 3, \pi / 3, \pi / 3,-2 \pi / 3)$ plus its 3 nontrivial permutations (see Eq. (18) ). When $\alpha>2 \pi / 3$ this zero bifurcates, and the distance between the two solutions grows with $\alpha$. These are the solutions whose explicit expressions are given in Eq. (24) of Sect. 5.

We want now to understand under what conditions other zeros besides the "standard" ones can appear when $\alpha_{\mu}$ is not restricted to be on a major hypercubic diagonal, and establish some bounds for the regions of minimal doubling.

\section{A.3 Uniform bounds in the general case}

The trace equations for the sines, (37), constrain the ratios of sines to have the same value

$$
R=\frac{\sin \left(p_{\mu}-\alpha_{\mu} / 2\right)}{\sin \left(\alpha_{\mu} / 2\right)}
$$

in all four spacetime directions. The ratios of cosines can then be expressed for any given choice of $R$ as

$$
\frac{\cos \left(p_{\mu}-\alpha_{\mu} / 2\right)}{\cos \left(\alpha_{\mu} / 2\right)}= \pm \sqrt{R^{2}+\frac{1-R^{2}}{\cos ^{2}\left(\alpha_{\mu} / 2\right)}}
$$

It is easy to see from the trace equations for the cosines, (39), that it is not possible for these ratios of cosines to be greater than 1 at the same time for all four spacetime directions, and neither is possible that they are all smaller than 1 in the four directions. The case in which these ratios of cosines are all equal to 1 gives the "standard" zeros. Thus, for the action to have extra zeros in addition to the "standard" ones there must be at least one direction in which the ratio of the cosines in Eq. (39) is (strictly) greater than 1, and also at least one direction in which the ratio is instead (strictly) smaller than 1.

Now, the fact that at least one ratio of cosines must be greater than 1 implies that $R^{2}<1$ (as can be seen for instance from Eq. (47)). But this in turn constrains those ratio of cosines that are smaller than 1 in the cosine equation (39) to the much 
stronger requirement of being actually smaller than -1 . This observation is crucial for the following.

Let us at this point try to establish a uniform bound of the kind

$$
\cos \left(\alpha_{\mu} / 2\right) \geq C
$$

for the regions where extra zeros cannot appear (so that minimal doubling is preserved). Since, as we noted before, for at least one direction the ratio of cosines in the cosine equation must be (strictly) smaller than 1, and then actually negative and (strictly) smaller than -1 , let us consider the case where this occurs for only one direction (while for the other three directions is instead positive and strictly greater than 1$)$. Then, -1 is the strict upper bound for this one negative term in the left hand side of the cosine equation (39), while Eq. (47) allows us to set an upper bound, which depends on $C$, for the sum of the other three terms.

Now, extra zeros can be certainly excluded if the left hand side of the cosine equation in this configuration remains always (strictly) smaller than 4, and this is the case if the inequality

$$
3 \sqrt{R^{2}+\frac{1-R^{2}}{C^{2}}} \leq 5
$$

holds. This is $C^{2} \geq\left(R^{2}-1\right) /\left(R^{2}-25 / 9\right)$, which gives the uniform bound $C \geq 3 / 5$ (attained when $R=0$ ). Thus, when for all directions

$$
\cos \left(\alpha_{\mu} / 2\right) \geq \frac{3}{5}
$$

which amounts to $\alpha_{\mu} \leq 0.590334 \pi \sim 106.26^{\circ}$, minimal doubling is guaranteed. It is easy to see that the remaining cases in which the ratios of cosines are negative for more than one direction cannot improve this bound.

We next prove that when for all directions the uniform bound

$$
\cos \left(\alpha_{\mu} / 2\right) \leq \frac{1}{2}
$$

is satisfied, then there are always extra zeros, and minimal doubling is lost. For this we will also use again some considerations from the previous subsection.

Using the expression (47) (which already contains the information from Eq. (37)), the cosine equation (39) can be rewritten as

$$
\sqrt{R^{2}+\frac{1-R^{2}}{\cos ^{2}\left(\alpha_{1} / 2\right)}}+\sqrt{R^{2}+\frac{1-R^{2}}{\cos ^{2}\left(\alpha_{2} / 2\right)}}+\sqrt{R^{2}+\frac{1-R^{2}}{\cos ^{2}\left(\alpha_{3} / 2\right)}}-\sqrt{R^{2}+\frac{1-R^{2}}{\cos ^{2}\left(\alpha_{4} / 2\right)}}=4,
$$

where we consider one of the possible choices of signs in front of the square roots which can give extra zeros (as discussed in the previous subsection).

Thanks to the symmetry under permutations we can always assume that $\cos \left(\alpha_{3} / 2\right) \leq$ $\cos \left(\alpha_{4} / 2\right) \cdot 8$

\footnotetext{
${ }^{8}$ Otherwise one has to consider a different equation, similar to (52) but where the negative sign is in front of another square root instead of the fourth one.
} 
We know from the previous subsection that when there are extra zeros it must also be $R^{2}<1$. When $R^{2}$ increases from 0 to 1 the value of each square root decreases monotonically, from its possible maximum $1 / \cos \left(\alpha_{\mu} / 2\right)$ to its possible minimum 1 . Moreover, because $\cos \left(\alpha_{3} / 2\right) \leq \cos \left(\alpha_{4} / 2\right)$ the difference of the third and fourth square roots must be nonnegative.

When $R=0$ the sum of the first and second square roots assumes its maximum possible value, which because of (51) has to be at least 4. Since the difference of the third and fourth square roots is nonnegative, the left hand side of (152) gives then a result which is always greater or equal to 4 , when $R=0$.

When $R^{2}=1$, the result of the left hand side of (152) assumes its possible minimum, which is exactly 2 (whatever the choice of $\alpha_{\mu}$ ).

Because of the monotonicity of each square root, and of the fourth square root being always smaller in absolute value than the third one (or at most equal to it), the left hand side of (52) will decrease monotonically when $R^{2}$ increases from $R^{2}=0$ (where its value it at least 4 ) towards $R^{2}=1$ (where its value is 2). Then there is always a value of $R^{2}$ for which the left hand side will become exactly equal to 4 . This demonstrates that extra zeros always exist if the bound (51) holds.

\section{References}

[1] M. Creutz, JHEP 0804 (2008) 017 [arXiv:0712.1201 [hep-lat]].

[2] A. Boriçi, Phys. Rev. D 78 (2008) 074504 arXiv:0712.4401 [hep-lat]].

[3] M. Creutz, PoS LATTICE 2008 (2008) 080 [arXiv:0808.0014 [hep-lat]].

[4] A. Boriçi, PoS LATTICE 2008 (2008) 231 [arXiv:0812.0092 [hep-lat]].

[5] T. Misumi, PoS LATTICE 2012 (2012) 005 arXiv:1211.6999 [hep-lat]].

[6] M. Creutz, arXiv:1308.3672 [hep-lat].

[7] L. H. Karsten, Phys. Lett. B104 (1981) 315.

[8] F. Wilczek, Phys. Rev. Lett. 59 (1987) 2397.

[9] S. Capitani, J. Weber and H. Wittig, Phys. Lett. B 681 (2009) 105 arXiv:0907.2825 [hep-lat]].

[10] S. Capitani, J. Weber and H. Wittig, PoS LATTICE 2009 (2009) 075 arXiv:0910.2597 [hep-lat]].

[11] S. Capitani, M. Creutz, J. Weber and H. Wittig, JHEP 1009 (2010) 027 arXiv:1006.2009 [hep-lat]].

[12] S. Capitani, M. Creutz, J. Weber and H. Wittig, PoS LATTICE 2010 (2010) 093 arXiv:1010.0110 [hep-lat]].

[13] M. Creutz, PoS LATTICE 2010 (2010) 078 [arXiv:1009.3154 [hep-lat]]. 
[14] M. Creutz, Acta Phys. Slov. 61 (2011) 1 [arXiv:1103.3304 [hep-lat]].

[15] S. Capitani, arXiv:1307.7497 [hep-lat].

[16] P. F. Bedaque, M. I. Buchoff, B. C. Tiburzi and A. Walker-Loud, Phys. Lett. B 662 (2008) 449 [arXiv:0801.3361 [hep-lat]]. 